\title{
Insulin-like Growth Factor 1, but Not Insulin-Like Growth Factor-Binding Protein 3, Predicts Central Precocious Puberty in Girls 6-8 Years Old: A Retrospective Study
}

\author{
Patricia Diaz Escagedo ${ }^{a, b} \quad$ Cheri L. Deal ${ }^{b, c}$ Andrew A. Dwyer ${ }^{d}$ \\ Michael Hauschild ${ }^{a, e}$
}

${ }^{a}$ Faculty of Biology and Medicine, University of Lausanne, Lausanne, Switzerland; ${ }^{b}$ Endocrine and Diabetes Service, CHU Sainte-Justine and University of Montreal, Montreal, QC, Canada; ${ }^{\circ}$ Research Center and Department of

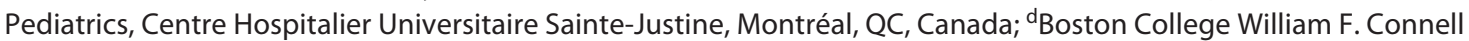
School of Nursing and Harvard Reproductive Endocrine Sciences Center, Boston, MA, USA; ePediatric Endocrinology, Diabetes and Obesity Unit, Service of Pediatrics, Department Woman-Mother-Child, Centre Hospitalier Universitaire Vaudois (CHUV), Lausanne, Switzerland

\section{Keywords \\ Central precocious puberty · Growth factors · Insulin-like growth factor-1}

\begin{abstract}
Background: Central precocious puberty (CPP) in females is characterized by thelarche before 8 years of age. Evidence of reproductive axis activation confirms the diagnosis (basal serum luteinizing hormone $(\mathrm{LH}) \geq 0.3 \mathrm{IU} / \mathrm{L}$ or $\mathrm{LH}$-releasing hormone (LHRH)-stimulated $\mathrm{LH} \geq 5 \mathrm{IU} / \mathrm{L})$. Stimulation testing is the diagnostic gold standard but is time-consuming and costly. Serum levels of insulin-like growth factor-1 (IGF-1) and insulin-like growth factor-binding protein 3 (IGFBP-3) are increased in girls with CPP. Objective: The aim of the study was to assess the utility of serum IGF-1 and IGFBP-3 in identifying CPP in girls aged 6-8 years. Methods: The study was a single-center retrospective study. Girls with confirmed CPP $(n=44)$ and isolated premature precocious adrenarche/ precocious thelarche (PA/PT, $n=16$ ) had baseline biochemical profiling and LHRH stimulation testing. Serum IGF-1 and IGFBP-3 results were converted to standard deviation scores
\end{abstract}

(SDS). Correlations were calculated and receiver operating characteristic curves were plotted. Results: Girls with CPP had higher basal and peak LH, IGF-1 SDS, and growth velocity $(p<0.05)$. IGF-1 SDS correlated positively with basal and peak LH ( $p<0.05)$. IGF-1 SDS (1.75-2.15) differentiated CPP and PA/PT with $89 \%$ sensitivity and $56 \%$ specificity (basal LH) and $94 \%$ specificity and $55 \%$ sensitivity (peak LH). IGFBP-3 SDS did not differ between groups or by CPP parameters. Conclusions: In clinical practice, IGF-1 SDS may be an additional tool for identifying CPP in girls aged 6 to 8 years when baseline clinical and laboratory diagnostic criteria are inconclusive, possibly avoiding more time-consuming and costly procedures.

(c) 2021 The Author(s)

Published by S. Karger AG, Basel

\section{Introduction}

Precocious puberty (PP) in female individuals is defined by the onset of breast development before the age of 8 years. Central PP (CPP) is confirmed by documenting hypothalamic-pituitary-gonadal (HPG) axis activation. karger@karger.com www.karger.com/hrp

Karger $\stackrel{\text { ! }}{\text { - }}$

BOPEN ACCESS
(C) 2021 The Author(s)

Published by S. Karger AG, Basel

This article is licensed under the Creative Commons Attribution 4.0 International License (CC BY) (http://www.karger.com/Services/ OpenAccessLicense). Usage, derivative works and distribution are permitted provided that proper credit is given to the author and the original publisher.
Correspondence to:

Michael Hauschild, michael.hauschild@ chuv.ch 
Notably, CPP is $15-20$ times more common in girls, and its incidence is increasing $[1,2]$. The etiology of CPP in girls is predominantly idiopathic (86\%) with an interaction of environmental and genetic (monogenic or polygenic) influences [3-6].

Pubertal onset in girls is marked by estrogen-dependent physical changes including breast development, accelerated growth velocity, and bone maturation. Activation of the HPG axis is marked by pulsatile gonadotropinreleasing hormone secretion that stimulates gonadotropin secretion. Increased serum luteinizing hormone (LH) levels trigger estradiol secretion. Concurrently, HPG axis activation amplifies growth hormone secretion from the anterior pituitary and increased hepatic production of insulin-like growth factor-1 (IGF-1) and its major circulating binding protein (insulin-like growth factor-binding protein 3, IGFBP-3). Estradiol stimulates growth hormone secretion centrally and peripherally, leading to increased local IGF-1 production/action in the epiphyseal growth plates. These hormonal dynamics underlie the pubertal growth spurt with increased growth velocity during Tanner II-III breast development and higher serum levels of IGF-1 and its binding protein IGFBP-3 [3, 7]. Girls with CPP have older bone age (BA) than IGF-1matched prepubertal girls $[8,9]$, and elevated serum IGF-1 levels reflect greater growth velocity - an early sign of puberty in girls [10].

Currently, serum IGF-1 levels are not considered part of the standard evaluation for CPP. It is important to differentiate CPP from isolated precocious thelarche (PT) or precocious adrenarche $(\mathrm{PA})$ as clinical evolution and treatment differ. Laboratory diagnostic criteria for CPP include basal serum LH concentrations $\geq 0.3 \mathrm{IU} / \mathrm{L}$ or LHreleasing hormone (LHRH)-stimulated serum $\mathrm{LH} \geq 5$ IU/L $[11,12]$. Detectable estradiol and ultrasound findings (i.e., uterine length $\geq 35 \mathrm{~mm}$ with signs of estrogenization, ovarian volume $\geq 2 \mathrm{~mL}$ ) also support the diagnosis $[5,12-14]$. Importantly, CPP may result in diminished adult height $(\mathrm{AH})$ as well as psychological distress related to body changes/precocious menarche. Accordingly, there is an ongoing debate regarding the treatment of girls with CPP [15]. Gonadotropin-releasing hormone analogs are considered standard of care for girls with pubertal onset before 6 years and can help patients gain up to $9-10 \mathrm{~cm}$ in final height [16]. However, in girls older than 6 years, the decision to initiate treatment is less clear and is typically made on a case-by-case basis. Notably, studies demonstrate that $1 / 3$ of nontreated girls between 6 and 8 years old have decreased $\mathrm{AH}$ and could therefore benefit from the therapy $[16,17]$.

IGF-1 Predicts Central Precocious

Puberty in 6- to 8-Year-Old Girls
Despite the known relationship between IGF-1 and IGFBP-3 levels and CPP, there is a paucity of data examining the use of growth factors (IGF-1 and IGFBP-3) to assist in diagnosing girls with CPP. We aimed to examine the utility of BA-corrected serum IGF-1 and IGFBP-3 levels at initial evaluation of CPP in girls 6-8 years when clinical and laboratory diagnostic findings are inconclusive.

\section{Methods}

This retrospective study included all girls with suspicion of CPP who were referred to the Lausanne Pediatric Endocrinology Clinic (1997-2017).

\section{Participants}

Diagnostic criteria for idiopathic CPP included Tanner $\geq \mathrm{II}$ breast development and basal serum $\mathrm{LH} \geq 0.3 \mathrm{IU} / \mathrm{L}$ or serum LHRH-stimulated serum $\geq 5 \mathrm{IU} / \mathrm{L}$. Girls with the onset of puberty $<6$ or $>8$ years as well as girls not meeting idiopathic CPP diagnostic criteria (i.e., peripheral CPP, abnormal brain MRI/tumor, cancer survivors) were excluded. Individuals with missing data (i.e., no LHRH stimulation test, no documented serum $\mathrm{LH} \geq 0.3 \mathrm{IU} / \mathrm{L}$, no documented serum IGF-1 or IGFBP-3) were excluded from the analysis. As a comparison group, data were collected from girls aged 6-8 years diagnosed with PA or PT. These patients were selected from the girls referred for evaluation of early pubertal onset, yet PP was ruled out and PA or PT was diagnosed. Specifically, PA was defined as $\geq$ Tanner II pubarche without thelarche, and PT was defined as $\geq$ Tanner II breast development with LHRH-stimulated serum LH $<5$ IU/L. Girls diagnosed with PT or PA lacking serum IGF-1 and IGFBP-3 measures were excluded from the analyses. Girls with growth factor measurements were included in the analyses. Girls were then classified as either CPP or PA/PT.

\section{Measures}

Familial and personal history, auxological parameters including patient height and weight, BMI, and their respective standard deviation scores (SDS) [18] were collected. Growth velocity was calculated using height at initial evaluation and follow-up (4-6 months without treatment). BA was assessed according to the Greulich Pyle atlas [19]. Stimulation testing was performed using 100 mcg LHRH (Relefact ${ }^{\circledR}$ ). Blood samples were collected at baseline, $+30,+45,+60$, and +90 min after IV bolus.

Assays

Serum LH (IU/L) and follicle stimulating hormone (FSH) (IU/L) were measured using AxSYM, the Abbott microparticle enzyme immunoassay (1997-June 2014; LH level of detection: 0.5 $\mathrm{IU} / \mathrm{L}$ and inter-assay coefficients of variation [CVs]: $6 \%$; FSH level of detection: $0.4 \mathrm{IU} / \mathrm{L}$ and inter-assay CVs: $6.7 \%$ ) or the Roche Cobas 8000 (module e602) electrochemiluminescence immunoassay (July 2014-2017, LH level of detection: 0.1 IU/L, intra-assay CVs: $0.9 \%$ and inter-assay CVs: $1.4 \%$; FSH level of detection: 0.1 IU/L, intra-assay CVs: $1.6 \%$ and inter-assay CVs: $1.7 \%$ ). Crossover studies conducted by the laboratory in 2014 demonstrated excellent positive correlation (LH: 0.99 and FSH: 0.97) providing a basis for considering the assays equivalent for comparisons. Dehydro- 


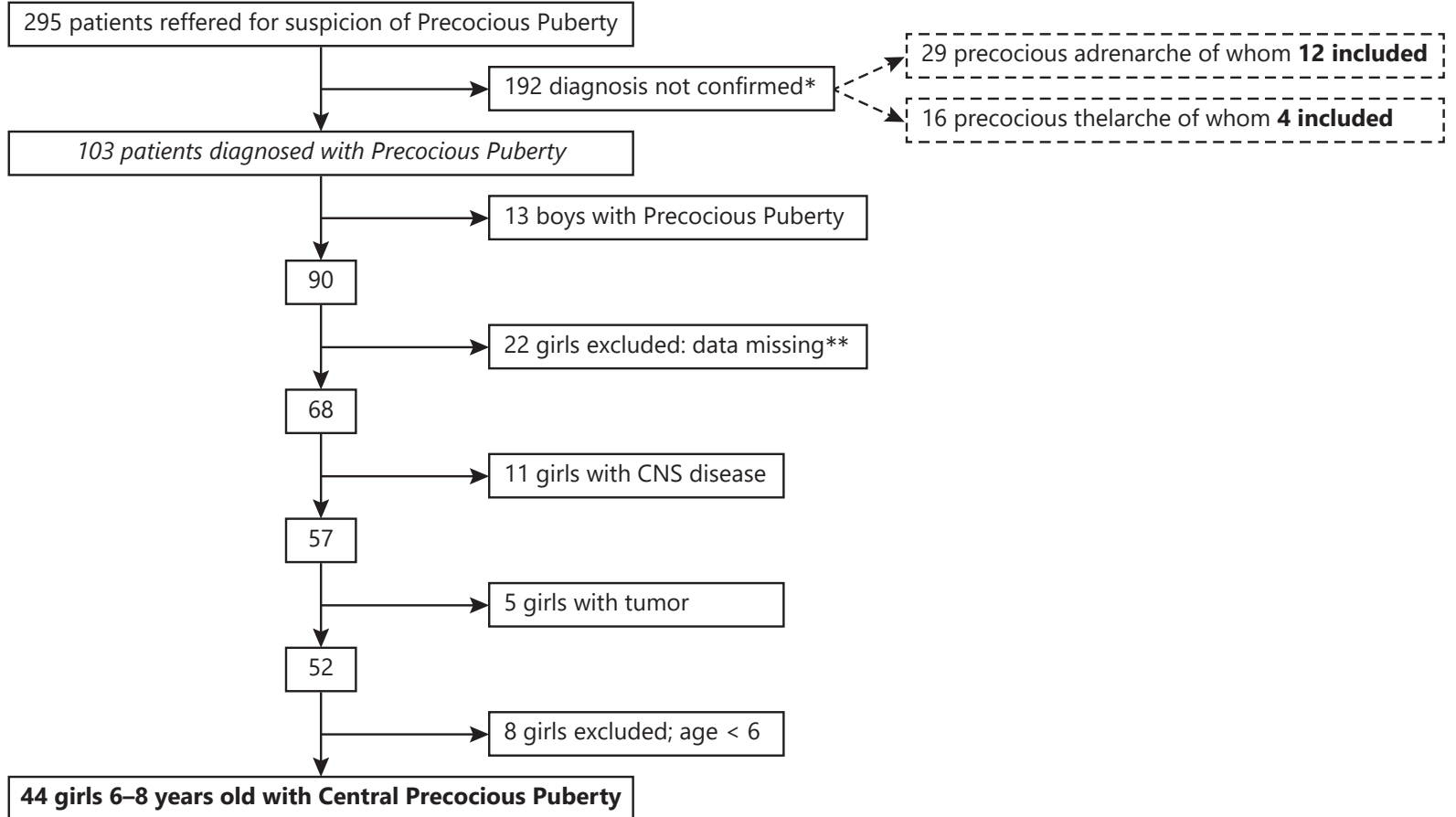

*29 PA (12 with IGF-1/IGFBP-3 measured), 16 PT (4 with IGF-1/IGFBP-3 measured), 8 CAH, 11 PPP, 16 boys, 61 referred >8.5 yo,

$51 \mathrm{LHRH}$ stimulation test missing

**No IGF-1/IGFBP-3

Fig. 1. Flowchart depicting retrospective identification of cases (1997-2017) from the pediatric endocrinology clinic. PA, precocious adrenarche; $\mathrm{PT}$, precocious thelarche; $\mathrm{CAH}$, congenital adrenal hyperplasia; PPP, peripheral precocious puberty; IGF-1, insulin-like growth factor 1; IGFBP-3, insulin-like growth factor-binding protein 3; PP, precocious puberty; CPP, central precocious puberty.

epiandrosterone sulfate (DHEAS $[\mu \mathrm{mol} / \mathrm{L}])$ levels were retrieved from the patient's charts. All laboratory measurements were done in the same laboratory. The methodology for measuring IGF-1 $(\mu \mathrm{g} / \mathrm{L})$ and IGFBP-3 $(\mathrm{mg} / \mathrm{L})$ changed during the 20 -year period. Initially, IGF-1 was measured using the Nichols Institute Diagnostics radioimmunoassay (NID-RIA) from 1997 until it was taken off the market in August 2005. Subsequently, the Immulite Siemens 2000 chemiluminescent enzyme immunometric assay was used (2005-2017). This assay was standardized to the World Health Organisation (WHO) 1st IRR 87/518 until January 2017- when it was restandardized to the WHO 1st IS 02/254. We measured IGFBP-3 using the NID-RIA (1997-September 2005). Thereafter, the Immulite Siemens 2000 chemiluminescent enzyme immunometric assay was employed and standardized to WHO 93/560 in March 2006 (no prior international standard). To harmonize results, we converted IGF-1 and IGFBP-3 results using SDS. Scores were corrected for sex and age (IGF-1 SDS and IGFBP-3 SDS) or sex and BA (BA IGF-1 SDS and BA IGFBP-3 SDS) [20, 21].

\section{Analysis}

Statistical analyses were performed using R (version 4.0.2). Data are reported descriptively using mean \pm SD and median (range) depending on the parameter and distribution. Continuous data were assessed for normality using distribution graphics and the Shapiro-Wilk Test. Student's $t$ test or the Mann-Whitney U test was used to compare groups, as appropriate. We employed Spearman's correlation to examine the correlation between the variables not normally distributed. Linear regression was performed to examine the associations between IGF-1 SDS/IGFBP-3 SDS and parameters defining CPP. Stepwise multivariate linear regression was performed to assess interactions between IGF-1 SDS, BMI SDS, BA to chronological age ratio (BA/CA), DHEAS, and basal LH. Receiver operating characteristics (ROC) curves were generated to determine whether IGF-1 SDS and IGFBP-3 SDS could discriminate between CPP and PA/PT. A $p$ value $<0.05$ was considered statistically significant.

\section{Results}

In total, 295 children were referred to the tertiary center between January 1997 and December 2017 for evaluation of early puberty (Fig. 1). We included for analysis 44 girls aged 6-8 years with idiopathic CPP as well as 16 who met 
Table 1. Clinical and biochemical characteristics of patients with CPP and PA/PT

\begin{tabular}{lclc}
\hline Characteristic & $\begin{array}{l}\text { CPP }(n=44) \text { mean } \pm \text { SD, } \\
n \text { or median }(\text { range })^{*}\end{array}$ & $\begin{array}{l}\text { PA/PT }(n=16) \text { mean } \pm \text { SD, } \\
n \text { or median (range) }\end{array}$ & $p$ value \\
\hline Thelarche/pubarche onset, years & $7.3(6-7.9)(44)$ & $7.1(6.5-7.9)(16)$ & 0.8 \\
BA, years & $9.7 \pm 1.2(44)$ & $9.2 \pm 1.1(16)$ & 0.17 \\
Tanner breast stage & $2.5(2-3)(44)$ & $2.2(1-3)(16)$ & $\mathbf{0 . 0 2}$ \\
Tanner pubarche stage & $2(1-4)(44)$ & $2(1-3)(16)$ & 0.52 \\
Height SDS & $2.1 \pm 1.1(44)$ & $2.1 \pm 1.2(16)$ & 0.9 \\
BMI SDS & $1.5 \pm 2(44)$ & $2.1 \pm 1.5(16)$ & 0.25 \\
Growth velocity, cm/year & $9.9 \pm 3.4(40)$ & $7.7 \pm 1.9(13)$ & $\mathbf{0 . 0 3}$ \\
Basal LH, IU/L & $1.1(0.1-7)(44)$ & $0.1(0.1-0.2)(15)$ & $<\mathbf{0 . 0 0 1}$ \\
Peak LH, IU/L & $13.2(5.3-44.4)(38)$ & $9.1 \pm 3(12)$ & 0.000001 \\
Peak FSH, IU/L & $11.6 \pm 4.6(38)$ & $1.5(0.4-4.8)(13)$ & 0.06 \\
DHEAS, $\mu$ mol/L & $0.8(0.1-3.2)(29)$ & $0.1(-1.5$ to 2$)(16)$ & $<\mathbf{0 . 0 0 1}$ \\
IGF-1 SDS & $1.8(-1.2$ to 3.5$)(44)$ & $0.6(-1.7$ to 1.5$)(16)$ & $<\mathbf{0 . 0 0 1}$ \\
BA IGF-1 SDS & $1.2(-1.4$ to 2.9$)(44)$ & $1.5(0.6-3.4)(15)$ & 0.95 \\
IGFBP-3 SDS & $1.35(-0.4$ to 3.4) $(42)$ & $1(0-3.4)(15)$ & 0.45 \\
BA IGFBP-3 SDS & $0.8(-0.4$ to 2.9$)(42)$ & & \\
\hline
\end{tabular}

SDS, standard deviation scores; LH, luteinizing hormone, FSH, follicle-stimulating hormone, DHEAS, dehydroepiandrosterone sulfate; IGF-1, insulin-like growth factor 1; IGFBP-3, insulin-like growth factor-binding protein 3; BA, bone age adjusted; PA, precocious adrenarche; PT, precocious thelarche; CPP, central precocious puberty. ${ }^{*}$ Median and range reported for non-Gaussian distributions.

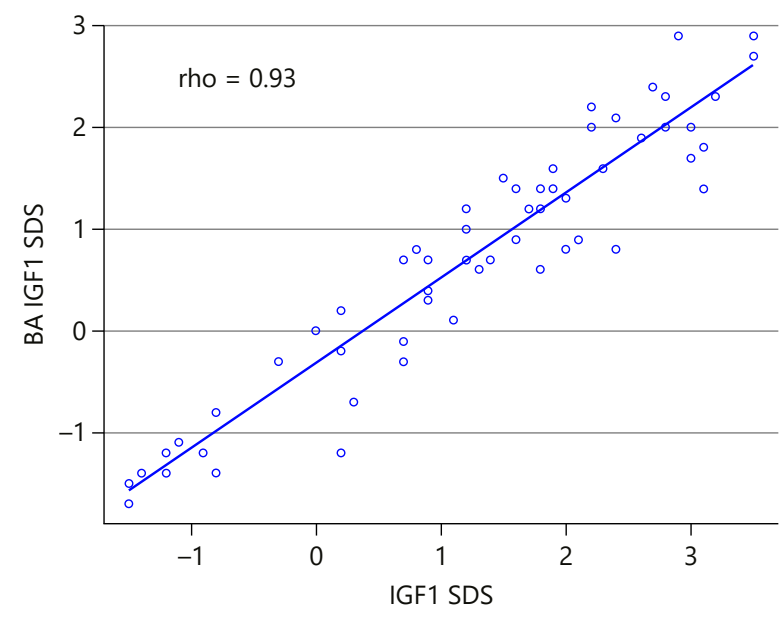

Fig. 2. Relationship between age-corrected IGF-1 SDS and scores corrected for BA. SDS, standard deviation scores; BA, bone age; IGF-1, insulin-like growth factor.

criteria for PA/PT, all of whom had IGF-1 and IGFBP-3 measured. Descriptive characteristics, demographic information, auxological parameters, and biochemical profiles are summarized in Table 1 . Our cohort was mostly White, 28/44 (63.7\%) for CPP and 13/16 (81.25\%) for PA/PT.
Other subjects were Hispanic, 5/44 (11.4\%) for CPP and 2/16 (12.5\%) for PA/PT; Asian, 4/44 (9\%); Black/African American, 2/44 (4.6\%); Pacific Islander, $1 / 44$ (2.3\%) and 4/44 (9\%) for CPP; and 1/16 (6.25\%) for PA/PT were multiracial [22]. The median age at the onset of secondary sexual characteristics was 7.3 years (CPP) and 7.1 years (PA/ PT) $(p=0.8)$. Eighteen percent of the CPP girls were adopted (none in the PA/PT group). Both groups exhibited advanced $\mathrm{BA}(\mathrm{CPP}=9.7$ vs. $\mathrm{PA} / \mathrm{PT}=9.2$ years, $p=0.17)$. Similarly, neither mean height nor mean BMI SDS differed between groups. As expected, Tanner breast stage, median basal LH, peak LH, IGF-1 SDS, and BA IGF-1 SDS differed between groups (all, $p<0.05$ ). Mean growth velocity was significantly higher in girls with CPP ( 9.9 vs. $7.7 \mathrm{~cm} /$ year, $p=0.02)$. Groups neither differed in IGFBP-3 SDS $(p=$ $0.95)$ nor BA IGFBP-3 SDS $(p=0.45)$.

\section{IGF- 1 and IGFBP-3 SDS}

We observed significant, positive correlations between IGF-1 SDS and BA IGF-1 SDS $(r=0.93, p<0.00001)$ as well as IGFBP-3 SDS and BA IGFBP-3 SDS $(r=0.86, p<$ $0.00001)$. The correlation remained significant after analyzing the CPP and PA/PT groups separately (IGF-1 SDS: $r=0.9$ and 0.95 , respectively, both $p<0.00001$; IGFBP-3 SDS: $r=0.86$ and 0.83 , respectively, both $p<0.0001$ ) (Fig. 2). Based on these findings, subsequent analyses were 
Table 2. Relationship between IGF-1 SDS and IGFBP-3 and CPP parameters

\begin{tabular}{lll}
\hline & $r^{2}$ & $p$ value \\
\hline IGF-1 SDS & & \\
Growth velocity & 0.1 & $\mathbf{0 . 0 2}$ \\
BMI SDS & 0.04 & 0.137 \\
BA/CA & 0.01 & $\mathbf{0 . 0 2}$ \\
Basal LH & 0.10 & $\mathbf{0 . 0 1}$ \\
Peak LH & 0.23 & $\mathbf{0 . 0 0 0 3}$ \\
DHEAS & 0.17 & $\mathbf{0 . 0 0 7}$ \\
IGFBP-3 & & \\
Growth velocity & 0.045 & 0.14 \\
BMI SDS & 0.0003 & 0.907 \\
BA/CA & 0.018 & 0.324 \\
Basal LH & 0.006 & 0.556 \\
Peak LH & 0.012 & 0.446 \\
DHEAS & 0.02 & 0.312 \\
\hline
\end{tabular}

BA, bone age; $\mathrm{CA}$, chronological age; $\mathrm{LH}$, luteinizing hormone; DHEAS, dehydroepiandrosterone sulfate; SDS, standard deviation scores; IGF-1, insulin-like growth factor 1; IGFBP-3, insulin-like growth factor-binding protein 3; CPP, central precocious puberty.

performed using IGF-1 SDS and IGFBP-3 SDS. Moreover, using SDS is an easier approach than BA-adjusted measures and is more reproducible in daily clinical practice.

\section{Simple Linear and Stepwise Multivariate Linear}

Regression

Simple linear regression showed that IGF-1 SDS is correlated with growth velocity $\left(r^{2}=0.1, p=0.02\right)$, BA/CA $\left(r^{2}=0.01, p=0.02\right)$, basal LH $\left(r^{2}=0.1, p=0.01\right)$, peak LH $\left(r^{2}=0.23, p=0.0003\right)$, and DHEAS $\left(r^{2}=0.17, p=0.007\right)$. No correlation was observed between IGF-1 SDS and BMI SDS. Similarly, there was no association between IGFBP-3 SDS and other CPP parameters (Table 2). Stepwise multivariate linear regression revealed a significant association between BA/CA and LHRH response ( $p=$ 0.02 ). Thus, each year of advanced BA increases the likelihood of a pubertal response to LHRH stimulation by 30 (Table 3).

\section{Cutoff Values Discriminating CPP and PA/PT}

ROC curve analysis revealed IGF-1 SDS cutoff values discriminating CPP and PA/PT. Youden's index was used to select the optimal IGF-1 SDS cutoff points for basal LH $(2.15,70 \%$ accurate $)$ and 1.75 for peak LH $(1.75,65 \%$ accurate) (Table 4). Comparing peak $\mathrm{LH}$ to basal $\mathrm{LH}$, IGF-1 SDS was better at discriminating CPP from PA/PT (peak LH area under the curve (AUC) $0.803 ; 95 \%$ confi-
Table 3. Stepwise multivariate linear regression analysis of factors associated with pubertal response (in GnRH stimulation test)

\begin{tabular}{llrl}
\hline LHRH response & $p$ value & OR & $95 \%$ CI \\
\hline IGF-1 SDS & 0.56 & 1.47 & $0.41-5.32$ \\
IGFBP-3 SDS & 0.96 & 1.05 & $0.16-7.11$ \\
BMI SDS & 0.18 & 2.31 & $0.68-7.91$ \\
BA/CA & $\mathbf{0 . 0 2}$ & 30.78 & $1.77-535.3$ \\
DHEAS & 0.21 & 0.39 & $0.09-1.7$ \\
\hline
\end{tabular}

LHRH, luteinizing hormone-releasing hormone; OR, odds ratio; CI, confidence interval; IGF-1, insulin-like growth factor 1; IGFBP-3, insulin-like growth factor-binding protein 3; SDS, standard deviation scores; $\mathrm{BA}$, bone age; CA, chronological age; DHEAS, dehydroepiandrosterone sulfate; GnRH, gonadotropinreleasing hormone.

dence interval: 0.683-0.922) (Fig. 3). This result was expected as basal LH is not always informative at the onset of puberty, particularly in light of earlier, less sensitive LH assays. The ROC curve analysis for IGFBP-3 did not discriminate between CPP and PA/PT (basal LH AUC 0.459; peak LH AUC 0.477). This result was not entirely unexpected as linear regression did not identify any correlations between IGFBP-3 and other puberty predictors.

\section{Discussion}

Currently, the LHRH stimulation test is the gold standard for diagnosing CPP. However, stimulation testing is time-consuming and costly. Herein, we examined the contribution of measuring growth factors (IGF-1 and IGFBP-3) in the initial CPP diagnostic workup of girls aged 6-8 years. Our data demonstrate that IGF-1 SDS, but not IGFBP-3 SDS, could be useful for discriminating true CPP from isolated, PA or PT. These findings are clinically relevant as many centers do not measure IGFBP-3 routinely.

To account for the large variability of IGF- 1 and IGFBP-3 during puberty, we used IGF-1 SDS and IGFBP-3 SDS corrected for CA, rather than BA. Both CA and BA have been used to minimize variability. However, from a clinical standpoint, it is more practical for clinical laboratories to calculate the IGF-1 SDS corrected for CA as opposed to BA.

Consistent with prior reports, we observed greater growth velocity, higher basal LH, peak LH, and IGF-1 SDS in girls with CPP than prepubertal peers. In the present study, IGFBP-3 was not informative. Prior work by Juul et al. showed that IGFBP-3 increases 2.5 -fold less than IGF-1
48

Horm Res Paediatr 2021;94:44-51 DOI: $10.1159 / 000516361$
Escagedo/Deal/Dwyer/Hauschild 
Table 4. IGF-1 SDS cutoff discriminating CPP and PA/PT

\begin{tabular}{|c|c|c|c|c|c|c|}
\hline & AUC (95\% CI) & Cutoff points & SS, $\%$ & SP, $\%$ & Accuracy, \% & Youden's index \\
\hline \multirow[t]{3}{*}{ Basal LH } & & -1.45 & 100 & 0 & & \\
\hline & 0.734 & 2.15 & 48 & 90 & 70 & 0.39 \\
\hline & $(0.608-0.86)$ & 3.35 & 0 & 100 & & \\
\hline \multirow[t]{3}{*}{ Peak LH } & & -1.45 & 100 & 0 & & \\
\hline & 0.803 & 1.75 & 55 & 94 & 65 & 0.48 \\
\hline & $(0.683-0.922)$ & 3.35 & 0 & 100 & & \\
\hline
\end{tabular}

AUC, area under the curve; CI, confidence interval; SS, sensitivity, SP, specificity; PA, precocious adrenarche; PT, precocious thelarche; CPP, central precocious puberty; SDS, standard deviation scores; LH, luteinizing hormone; IGF-1, insulin-like growth factor-1.
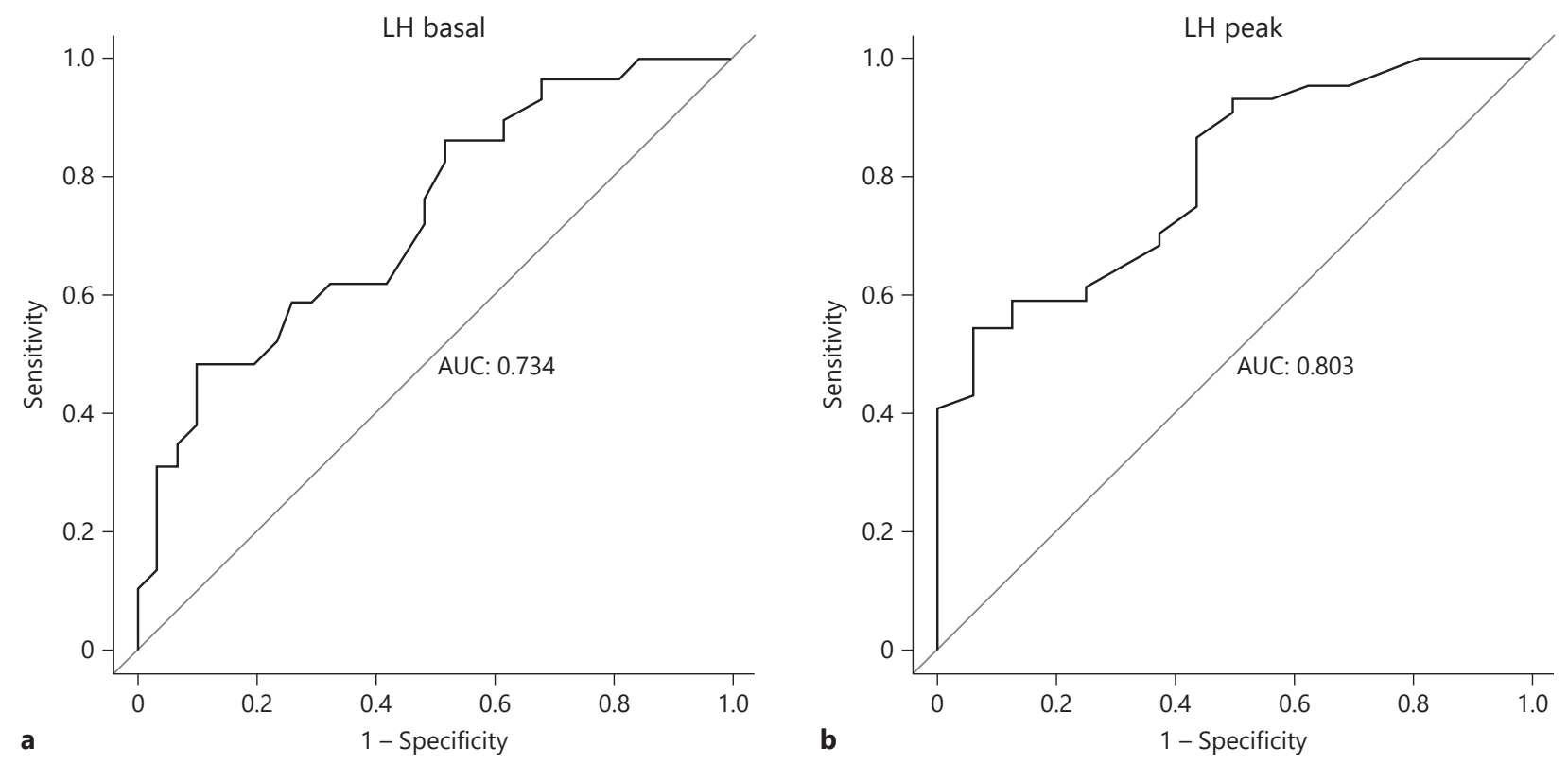

Fig. 3. ROC curves for IGF-1 SDS discriminating CPP and PA/PT. IGF-1 SDS and basal LH (a) and peak LH (b). AUC, area under the curve; ROC, receiver operating characteristic; IGF-1, insulin-like growth factor; SDS, standard deviation scores; PA, precocious adrenarche; PT, precocious thelarche; CPP, central precocious puberty; LH, luteinizing hormone.

during puberty [23]. Moreover, Sorensen et al. [8] found that IGFBP-3 corrected for BA did not differ between girls with CPP/early puberty and healthy controls. In our study, the IGFBP-3 SDS ( \pm 2 SD) using NID-RIA results overlapped with and were higher in girls aged 6-8 years than their 8- to 10-year-old counterparts. This observation may explain the similarity in CPP and PA/PT scores and the lack of correlation between IGFBP-3 SDS and PP parameters.
IGF-1 SDS did not correlate with BMI SDS, as already reported in other studies [24,25]. This finding was somewhat surprising because girls with a higher BMI are more likely to exhibit CPP $[8,26]$. Our cohort was not obese, and the BMI standard deviation was smaller than that in the population at large, perhaps explaining the absence of a correlation with IGF-1 SDS. 
We observed a positive correlation between IGF-1 SDS and classical parameters of CPP (i.e., growth velocity, BA/CA, basal LH, and peak LH). These findings are consistent with Sorensen et al. [8] and Juul et al. [27, 28], who reported higher IGF-1 levels in girls with CPP than aged-matched healthy girls. The ROC analysis showed that IGF-1 SDS can discriminate CPP and PA/ PT. We posit that IGF-1 SDS between 1.75 and 2.15 can be informative for differentiating $\mathrm{CPP}$ and $\mathrm{PA} / \mathrm{PT}$ in girls aged $6-8$ years. Thus, IGF-1 SDS may be an additional test when basal LH is still undetectable in girls aged 6-8 years who present with thelarche, increased growth velocity, and BA/CA >1 year. Importantly, measuring serum IGF-1 is less costly than LHRH stimulation testing.

A relative strength of this study is the number of 6- to 8 -year-old girls with CPP, which is the most difficult group considering treatment choices and the single-center approach. Moreover, the other studies previously cited included fewer patients, and inclusion criteria extended to 9-year-old girls $[8,28]$. However, the study has several limitations. First, a retrospective design can only infer associations. Second, assay methodology changed during the 20 -year period of included cases. We attempted to mitigate potential bias by normalizing the results with SD scores. Another limitation of this study is the lack of estradiol values, as well as AMH or inhibin $B$ values. However, as the study included patients seen as far back as 1997, many patients did not have estradiol measured as assays were less sensitive, particularly at the low end of the measurement. Finally, the PA/PT group has a limited number of patients because growth factors were not routinely assessed in these otherwise healthy patients.

To address these shortcomings, a prospective multicenter study including a larger cohort of patients and harmonized assays with single international standards would be required. This would also entail measuring IGF-1 levels regularly (i.e., every 6 months) to study association with growth velocity and longitudinal follow-up until adult age. To date, only one study $(n=23)$ has examined IGF-1 trends in girls with CPP compared with healthy controls [8]. This study did not identify any relationship with growth velocity or growth spurt.

\section{Conclusion}

Our study suggests that SDS IGF-1 may be a useful additional tool for identifying CPP in girls aged 6 to 8 years when baseline clinical and laboratory diagnostic criteria are inconclusive. Specifically, SDS IGF-1 may help discriminate $\mathrm{CPP}$ and $\mathrm{PA} / \mathrm{PT}$, thereby possibly avoiding more costly and time-consuming procedures. We did not observe any role for IGFBP-3 in the initial workup of CPP. Further prospective studies are needed to confirm our findings.

\section{Acknowledgements}

We thank Dominique Wagner for her assistance in calculating IGF-1 and IGFBP-3 SDS, the patients and their families, as the caregivers who took care of the patients. We also thank Jérôme Pasquier for his help in the statistics conception of the study.

\section{Statement of Ethics}

The study was conducted according to the principles outlined in the Declaration of Helsinki. The study (protocol \#2018-02123) was reviewed by the Commission Cantonale d'éthique de la recherche sur l'être humain which is the Institutional Review Board (Ethics Committee) of the University of Lausanne. Based on existing ethics statutes (art. $34 \mathrm{LRH}$ ), the study was deemed exempt from informed consent requirements as the study used retrospective deidentified data, did not modify the diagnosis or management of patients, and recontacting parents of children who no longer being followed was deemed unduly burdensome.

\section{Conflict of Interest Statement}

Cheri L. Deal is a member of the Editorial Board of Hormone Research in Paediatrics but will not participate as editor reviewer for the current article. The other authors have nothing to disclose.

\section{Funding Sources}

This research did not receive any specific grant from any funding agency in the public, commercial, or not-for-profit sector.

\section{Author Contributions}

P.D.E. and M.H. did the concept and design of the study. P.D.E. did the acquisition of the data and drafted the manuscript. P.D.E. and C.L.D. did the statistical analysis and interpretation of the data. A.A.D. helped with the interpretation of the data. All authors critically revised the manuscript for important intellectual content and approved the manuscript.
Escagedo/Deal/Dwyer/Hauschild 


\section{References}

1 Teilmann G, Pedersen CB, Jensen TK, Skakkebaek NE, Juul A. Prevalence and incidence of precocious pubertal development in Denmark: an epidemiologic study based on national registries. Pediatrics. 2005;116(6): 1323-8.

2 Bridges NA, Christopher JA, Hindmarsh PC, Brook CG. Sexual precocity: sex incidence and aetiology. Arch Dis Child. 1994;70(2): 116-8.

3 Wood CL, Lane LC, Cheetham T. Puberty: normal physiology (brief overview). Best Pract Res Clin Endocrinol Metab. 2019;33(3): 101265.

4 Leka-Emiri S, Chrousos GP, Kanaka-Gantenbein $\mathrm{C}$. The mystery of puberty initiation: genetics and epigenetics of idiopathic central precocious puberty (ICPP). J Endocrinol Invest. 2017;40(8):789-802.

5 Carel JC, Leger J. Clinical practice. Precocious puberty. N Engl J Med. 2008;358(22):236677.

6 Canton APM, Seraphim CE, Brito VN, Latronico AC. Pioneering studies on monogenic central precocious puberty. Arch Endocrinol Metab. 2019;63(4):438-44.

7 Perry RJ, Farquharson C, Ahmed SF. The role of sex steroids in controlling pubertal growth. Clin Endocrinol. 2008;68(1):4-15.

8 Sorensen K, Aksglaede L, Petersen JH, Andersson AM, Juul A. Serum IGF1 and insulin levels in girls with normal and precocious puberty. Eur J Endocrinol. 2012;166(5):903-10.

9 Harris DA, Van Vliet G, Egli CA, Grumbach MM, Kaplan SL, Styne DM, et al. Somatomedin- $\mathrm{C}$ in normal puberty and in true precocious puberty before and after treatment with a potent luteinizing hormone-releasing hormone agonist. J Clin Endocrinol Metab. 1985; 61(1):152-9.

10 Varimo T, Huttunen H, Miettinen PJ, Kariola L, Hietamaki J, Tarkkanen A, et al. Precocious puberty or premature thelarche: analysis of a large patient series in a single tertiary center with special emphasis on 6- to 8-year-old girls. Front Endocrinol. 2017;8:213.

11 Houk CP, Kunselman AR, Lee PA. Adequacy of a single unstimulated luteinizing hormone level to diagnose central precocious puberty in girls. Pediatrics. 2009;123(6):e1059-63.
12 Mogensen SS, Aksglaede L, Mouritsen A, Sorensen K, Main KM, Gideon P, et al. Diagnostic work-up of 449 consecutive girls who were referred to be evaluated for precocious puberty. J Clin Endocrinol Metab. 2011;96(5): 1393-401.

13 Kaplowitz PB. Do 6-8 year old girls with central precocious puberty need routine brain imaging? Int J Pediatr Endocrinol. 2016; 2016:9.

14 de Vries L, Horev G, Schwartz M, Phillip M. Ultrasonographic and clinical parameters for early differentiation between precocious puberty and premature thelarche. Eur J Endocrinol. 2006;154(6):891-8.

15 Kaplowitz PB, Backeljauw PF, Allen DB. Toward more targeted and cost-effective gonadotropin-releasing hormone analog treatment in girls with central precocious puberty. Horm Res Paediatr. 2018;90(1):1-7.

16 Carel JC, Eugster EA, Rogol A, Ghizzoni L, Palmert MR; Group, et al. Consensus statement on the use of gonadotropin-releasing hormone analogs in children. Pediatrics. 2009;123(4):e752-62.

17 Léger J, Reynaud R, Czernichow P. Do all girls with apparent idiopathic precocious puberty require gonadotropin-releasing hormone agonist treatment? J Pediatr. 2000;137(6):81925.

18 Sempé M, Pédron G, Roy-Pernot MJ. Auxologie, méthode et séquences. Paris: Théraplix; 1979. Vol. 93; p. 205.

19 Greulich WW, Pyle SI. Radiographic atlas of skeletal development of the hand and wrist. Stanford university press; 1959.

20 Le Bouc Y. [Insulin-like growth factors (IGF) and their binding proteins (IGFBP). Physiological and pathological regulation]. Journ Annu Diabetol Hotel Dieu. 1995:29-38.

21 Elmlinger MW, Kuhnel W, Weber MM, Ranke MB. Reference ranges for two automated chemiluminescent assays for serum insulinlike growth factor I (IGF-I) and IGF-binding protein 3 (IGFBP-3). Clin Chem Lab Med. 2004;42(6):654-64.
22 Institute of Medicine Subcommittee on Standardized Collection of Race/Ethnicity Data for Healthcare Quality Improvement; Ulmer C, McFadden B, Nerenz DR, editors. Race, ethnicity, and language data: standardization for health care quality improvement. Washington, DC: National Academies Press (US) Copyright 2009 by the National Academy of Sciences. All rights reserved; 2009.

23 Juul A, Dalgaard P, Blum WF, Bang P, Hall K, Michaelsen KF, et al. Serum levels of insulinlike growth factor (IGF)-binding protein-3 (IGFBP-3) in healthy infants, children, and adolescents: the relation to IGF-I, IGF-II, IGFBP-1, IGFBP-2, age, sex, body mass index, and pubertal maturation. J Clin Endocrinol Metab. 1995;80(8):2534-42.

24 Juul A, Bang P, Hertel NT, Main K, Dalgaard $\mathrm{P}$, Jorgensen $\mathrm{K}$, et al. Serum insulin-like growth factor-I in 1,030 healthy children, adolescents, and adults: relation to age, sex, stage of puberty, testicular size, and body mass index. J Clin Endocrinol Metab. 1994; 78(3):744-52.

25 Park MJ, Kim HS, Kang JH, Kim DH, Chung CY. Serum levels of insulin-like growth factor (IGF)-I, free IGF-I, IGF binding protein (IGFBP)-1, IGFBP-3 and insulin in obese children. J Pediatr Endocrinol Metab. 1999; 12(2):139-44.

26 Parent AS, Teilmann G, Juul A, Skakkebaek NE, Toppari J, Bourguignon JP. The timing of normal puberty and the age limits of sexual precocity: variations around the world, secular trends, and changes after migration. Endocr Rev. 2003;24(5):668-93.

27 Juul A, Fisker S, Scheike T, Hertel T, Muller J, Orskov H, et al. Serum levels of growth hormone binding protein in children with normal and precocious puberty: relation to age, gender, body composition and gonadal steroids. Clin Endocrinol. 2000;52(2):165-72.

28 Juul A, Scheike T, Nielsen CT, Krabbe S, Muller J, Skakkebaek NE. Serum insulin-like growth factor I (IGF-I) and IGF-binding protein 3 levels are increased in central precocious puberty: effects of two different treatment regimens with gonadotropin-releasing hormone agonists, without or in combination with an antiandrogen (cyproterone acetate). J Clin Endocrinol Metab. 1995;80(10):3059_ 67.
IGF-1 Predicts Central Precocious Puberty in 6- to 8-Year-Old Girls
Horm Res Paediatr 2021;94:44-51 DOI: $10.1159 / 000516361$ 